\title{
PIERRE FABRIE
}

\section{Solutions fortes et majorations asymptotiques pour le modèle de Darcy Forchheimer en convection naturelle}

\author{
Annales de la faculté des sciences de Toulouse $5^{e}$ série, tome 10, \\ $\mathrm{n}^{\circ} 1$ (1989), p. 7-26 \\ $<$ tttp://www.numdam.org/item?id=AFST_1989_5_10_1_7_0>
}

(C) Université Paul Sabatier, 1989, tous droits réservés.

L'accès aux archives de la revue «Annales de la faculté des sciences de Toulouse » (http://picard.ups-tlse.fr/ annales/) implique l'accord avec les conditions générales d'utilisation (http://www.numdam.org/conditions). Toute utilisation commerciale ou impression systématique est constitutive d'une infraction pénale. Toute copie ou impression de ce fichier doit contenir la présente mention de copyright.

\section{Numdam}

Article numérisé dans le cadre du programme

Numérisation de documents anciens mathématiques

http://www.numdam.org/ 


\title{
Solutions fortes et majorations asymptotiques pour le modèle de Darcy Forchheimer en convection naturelle
}

\author{
Pierre FABrie ${ }^{(1)}(2)$
}

RÉsumé. - Dans cet article nous établissons l'existence de solutions régulières uniformément bornées en temps pour un problème aux dérivées partielles fortement non linéaire modélisant la convection naturelle en milieu poreux. Ce modèle a été formulé sous cette forme par NIELDS D.A. et JOSEPH D.D. en 1985 [23]; il s'obtient en couplant l'équation de l'énergie et l'équation de Darcy-Forchheimer. Dans cet article on se limite au cas où le domaine $\Omega$ est un parallélépipéde de $\mathbf{R}^{3}$; en effet, dans ce cas, la solution de l'équation de Darcy-Forchheimer est régulière [11]. Le théorème principal de cet article établit une estimation $L^{\infty}\left(\mathbf{R}^{+} ; H^{1}(\Omega)\right)$ pour la température et une estimation $L^{\infty}\left(\mathbf{R}^{+} ;\left(\mathbf{H}^{1}(\Omega)\right)^{3}\right)$ pour la vitesse.

Abstract. - We discuss a strongly nonlinear system of partial differential equations which describes natural convection in porous medium. This model was given by NIELDES D.A. and JOSEPH D.D. in [23] 1985 ; it is obtained by coupling the energy equation and the Darcy-Forchheimer one. In this work we study only the case where the domain is a rectangular parallelepiped in $\mathbf{R}^{\mathbf{3}}$. In fact, in this case the solution of Darcy-Forchheimer equation is regular. The main result of this work is an $L^{\infty}\left(\mathbf{R}^{+} ; H^{1}(\Omega)\right)$ estimation of temperature and an $L^{\infty}\left(\mathbf{R}^{+} ;\left(H^{1}(\Omega)\right)^{3}\right)$ estimation of velocity.

\section{Introduction}

Nous étudions ici un système d'équations aux dérivées partielles qui modélise la convection naturelle dans un milieu poreux soumis à un gradient de température $\nabla T$.

(1) Centre de Recherche en Mathématiques de Bordeaux, Unité associée au CNRS 226, Université de Bordeaux 1, 351, Cours de la Libération, 33405 Talence

(2) Laboratoire Energétique et Phénomènes de Transfert, Unité associée au CNRS 873 


\section{P. Fabrie}

$\mathrm{Si}$, pour des nombres de Reynolds de pores inférieurs à l'unité, l'écoulement de convection naturelle est bien représenté par la loi de Darcy, au contraire, dans le cas de nombres de Reynolds plus élevés des phénomènes d'inertie doivent être pris en compte sous la forme d'un terme quadratique supplémentaire dans l'équation du mouvement. Les équations représentatives du phénomène dans le cas d'un fluide incompressible mais dilatable, $(\partial \rho / \partial p=0)$ s'écrivent :

$$
\left\{\begin{array}{l}
(\rho c)^{*} \frac{\partial T}{\partial t}-\operatorname{div}(\lambda \operatorname{grad} T)+(\rho c)_{f} V \cdot \operatorname{grad} T=0 \\
\rho \varepsilon^{-1} \frac{\partial V}{\partial t}+\mu K^{-1} V+c_{j} \rho K^{-1}|V| V+\operatorname{grad} \pi-\rho g=0 \\
\varepsilon \frac{\partial \rho}{\partial t}+\operatorname{div} \rho V=0 .
\end{array}\right.
$$

La première équation traduit la conservation de l'énergie, la seconde la conservation de la quantité de mouvement. Dans ce système, $\rho$ désigne la masse volumique, $V=\left(V_{1}, V_{2}, V_{3}\right)$ la vitesse de filtration, $\pi$ la pression, $T$ la température du milieu, $\varepsilon$ la porosité et $\mu$ la viscosité. $K$ et $\lambda$ sont les tenseurs respectifs de perméabilité et de conductivité thermique. La chaleur volumique du fluide est notée $(\rho c)_{f}$, celle du solide $(\rho c)_{s}$ et on définit alors la chaleur volumique équivalente par la relation $(\rho c)^{*}=\varepsilon(\rho c)_{f}+(1-\varepsilon)(\rho c)_{s}$. $c_{j}$ est une constante adimensionnelle.

De façon très classique dans les phénomènes de convection on simplifie ce modèle en faisant l'approximation de Boussinesq qui consiste à négliger les variations de la masse volumique, sauf dans le terme moteur $\rho g$; voir par exemple [4].

On se limitera ici au cas d'un milieu homogène isotrope remplissant une cavité parallélépipédique rectangle $\Omega$ dont l'un des axes est parallèle à l'accélération de la pesanteur $g$. Sous forme adimensionnelle le système s'écrit :

$$
\left\{\begin{array}{l}
\frac{\partial T}{\partial t}-\Delta T+V \cdot \nabla T=0 \\
\varepsilon^{-1} D a P_{r}^{*-1} \frac{\partial V}{\partial t}+V+c_{j} D a^{1 / 2} P_{r}^{*-1}|V| V+\nabla \pi+R a^{*} k t=0 \\
\operatorname{div} V=0
\end{array}\right.
$$

où $k$ est le vecteur unitaire porté par l'axe de la pesanteur. Outre la porosité $\varepsilon$ du milieu, il apparaît trois paramètres adimensionnels :

$$
\begin{aligned}
R a^{*} & =g \alpha\left(T_{2}-T_{1}\right) K(\rho c)_{f} \lambda^{-1} \rho \mu^{-1} L \\
P_{r}^{*} & =\mu \rho^{-1}(\rho c)^{*} \lambda^{*-1} \\
D a & =K L^{-2}
\end{aligned}
$$


$\left(T_{2}-T_{1}\right)$ est l'écart maximum de température dans le milieu et $L$ une longueur caractéristique.

Dans l'ouvert $\Omega=\{(x, y, z) \in] 0, L[\times] 0, \ell[\times] 0, h[\}$ de frontière $\Gamma$, les conditions aux limites rendant compte des parois adiabatiques et isothermes sont :

$$
\begin{gathered}
T(x, y, 0)=1, T(x, y, h)=0 \\
\frac{\partial T}{\partial x}(0, y, z)=\frac{\partial T}{\partial x}(1, y, z)=\frac{\partial T}{\partial y}(x, 0, z)=\frac{\partial T}{\partial y}(x, \ell, z)=0
\end{gathered}
$$

$V \cdot \nu_{\left.\right|_{\Gamma}}=0$ où $\nu$ est la normale unitaire sortante à $\Gamma$.

Nous noterons désormais $S=\varepsilon^{-1} D a P_{r}^{*-1}$ et $J=c_{j} D a^{1 / 2} P_{r}^{*-1}$.

On se ramène à des conditions aux limites homogènes en posant $T=$ $\theta+1-z / h$. Le système que nous étudions s'écrit pour $\sigma>1$ :

$$
\mathcal{P}^{\sigma} \begin{cases}(1.1) & \frac{\partial \theta}{\partial t}-\Delta \theta+V \cdot \nabla \theta-h^{-1} v_{3}=0 \\
(1.3) \quad & \theta(0 ; .)=\theta_{0} \\
& {\left[\begin{array}{c}
\theta(t ; x, y, 0)=\theta(t ; x, y, h)=0 \\
\frac{\partial \theta}{\partial x}(t ; 0, y, z)=\frac{\partial \theta}{\partial x}(t ; 1, y, z)=0 \\
\frac{\partial \theta}{\partial y}(t ; x, 0, z)=\frac{\partial \theta}{\partial y}(t ; x, \ell, z)=0
\end{array}\right.} \\
(1.5) \quad\left[\begin{array}{ll}
S \frac{\partial V}{\partial t}+V+J|V|^{\sigma-1} V+\nabla \pi+R a^{*} k \theta=0 \\
(1.6) \quad V(0 ; \cdot)=V_{0}
\end{array}\right. \\
(1.7) \quad V \cdot \nu_{\mid \mathrm{r}}=0\end{cases}
$$

Le cas $\sigma=2$ correspond au modèle physique présenté ci-dessus.

\section{Énoncés des résultats}

Introduisons tout d'abord quelques notations :

$\Omega=] 0, L[\times] 0, \ell[\times] 0, h[$

$\Gamma=\partial \Omega$ frontière de $\Omega, \Gamma^{*}=\Gamma \backslash\{$ arêtes $\}$

$\Gamma_{z}$ désigne la réunion des deux faces du parallélépipède d'équations $z=0$ et $z=h$ 


\section{P. Fabrie}

$\Gamma_{x y}$ désigne le manteau; il est défini par $\Gamma_{x y}=\Gamma \backslash \Gamma_{z}$

$H=\left\{\theta, \theta \in H^{1}(\Omega), \theta_{\mid \Gamma_{z}}=0\right\} ; \quad H^{\prime}$ le dual de $H$

$L^{P}=L^{P}(\Omega) ; \quad L^{P}=\left(L^{P}\right)^{3}$

pour $1<\sigma<\infty$ on pose :

$W^{\sigma}=\left\{V, V \in L^{\sigma} ; \operatorname{div} V=0, \gamma_{n}(V)=0\right\}$ où $\gamma_{n}$ est l'application linéaire définie de $W^{\sigma}(\operatorname{div})=\left\{V ; V \in L^{\sigma}\right.$, div $\left.V \in L^{\sigma}\right\}$ dans $W\left(\Gamma^{*}\right)^{-1 / \sigma, \sigma}$ qui prolonge l'application $V \rightarrow V \cdot \nu_{\Gamma^{*}}([14]$, lemme 1)

La formulation variationnelle du problème $\mathcal{P}^{\sigma}$ permet de préciser la notion de solution :

DÉFINITION 1.- Nous appellerons solution faible sur $[0, T]$ du problème $\mathcal{P}^{\sigma}$ un couple $(\theta, V)$ défini par :

$\theta \in \mathcal{C}^{0}\left(0, T ; L^{2}\right) \cap L^{2}(0, T ; H) ; \dot{\theta} \in L^{2}\left(0, T ; H^{\prime}\right)$

$V \in \mathcal{C}^{0}\left(0, T ; W^{2}\right) \cap L^{\sigma+1}\left(0, T ; W^{\sigma+1}\right) ; \dot{V} \in L^{1+1 / \sigma}\left(0, T ; W^{1+1 / \sigma}\right)$

$\forall \varphi \in L^{2}(0, T ; H)$

$\left.\int_{0}^{t}\langle\theta, \varphi\rangle d \tau+\int_{0}^{t} \int_{\Omega}(\nabla \theta \cdot \nabla \varphi) d \omega\right) d \omega d \tau+\int_{0}^{t}\langle B(V, \theta), \varphi\rangle d \tau$ $-h^{-1} \int_{0}^{t} \int_{\Omega} v_{3} \varphi d \omega d \tau=0$.

$\forall W \in L^{\sigma+1}\left(0, T ; W^{\sigma+1}\right)$

$S \int_{0}^{t} \int_{\Omega}(\dot{V} \cdot W) d \omega d \tau+\int_{0}^{t} \int_{\Omega}\left(V+J|V|^{\sigma-1} V \cdot W\right) d \omega d \tau+$

$R a^{*} \int_{0}^{t} \int_{\Omega}(k \theta \cdot W) d \omega d \tau=0$.

Nous démontrons dans cet article les deux résultats suivants :

ThÉORÈme 1.- Pour $\theta_{0}$ donné dans $L^{\infty}, 0 \leq \theta+1-z / h \leq 1$ et $V_{0}$ donné dans $W^{2}$ le problème $\mathcal{P}^{\sigma}$ admet une solution faible unique vérifiant:

$$
\theta(0 ; .)=\theta_{0} \quad \text { et } \quad V(0 ; .)=V_{0} .
$$

De plus $0 \leq \theta+1-z / h \leq 1$ presque partout.

Pour des données plus régulières la solution est plus régulière et vérifie les propriétés uniformes en temps précisées ci-dessous :

THÉORÈme 2.- Si $\theta_{0}$ est donné dans $H \cap L^{\infty}, 0 \leq \theta_{0}+1-z / h \leq 1$ et si $V_{0}$ est donné dans $W^{2} \cap\left(H^{1}(\Omega)\right)^{3}$ la solution du problème $\mathcal{P}^{\sigma}$ donnée 
au théorème 1 vérifie en outre les propriétés de régularité et de majoration asymptotiques suivantes :

$$
\begin{gathered}
\theta \in L^{\infty}\left(\mathbf{R}^{+} ; H\right) \cap L_{l o c}^{2}\left(\mathbf{R}^{+} ; H^{2}\right) \\
V \in L^{\infty}\left(\mathbf{R}^{+} ;\left(H^{1}(\Omega)\right)^{3}\right) \cap L_{l o c}^{\sigma+1}\left(\mathbf{R}^{+} ; W^{\sigma+1}\right) \\
\dot{\theta} \in L_{l o c}^{2}\left(\mathbf{R}^{+} ; L^{2}\right) .
\end{gathered}
$$$$
\text { De plus } 0 \leq \theta+1-z / h \leq 1 \text { presque partout. }
$$

Remarque 1. - i) : Avec $\theta_{0}$ donné dans $L^{2}$ et $V_{0}$ donné dans $W^{2}$ on peut définir une solution plus faible du problème $\mathcal{P}^{\sigma}$ où :

$$
\begin{aligned}
& \theta \in L^{\infty}\left(0, T ; L^{2}\right) \cap L^{2}(0, T ; H) \\
& \dot{\theta} \in L^{2}\left(0, T ;\left(H \cap H^{3 / 2}(\Omega)\right)^{\prime}\right) .
\end{aligned}
$$

On ne sait pas montrer l'unicité globale de telles solutions.

ii) : Sous l'hypothèse du théorème 1 on ne peut pas définir de solutions fortes car la condition de Neuman est vérifiée en un sens faible.

iii) : Sous les hypothèses du théorème 2 on peut écrire :

$$
\begin{array}{cc}
\dot{\theta}-\Delta \theta+V \cdot \nabla \theta-h^{-1} v_{3}=0 & \text { p.p. dans } \mathbf{R}^{+} \times \Omega \\
S V+\dot{V}+J|V|^{\sigma-1} V+\nabla \pi+R a^{*} k \theta=0 & \text { p.p. dans } \mathbf{R}^{+} \times \Omega \\
\operatorname{div} V=0 & \text { p.p. dans } \mathbf{R}^{+} \times \Omega \\
V(t ; .) \cdot \nu_{\left.\right|_{\Gamma}}=0 & \text { p.p. dans } \mathbf{R}^{+} \\
\theta(t ; .)_{\left.\right|_{\Gamma_{z}}}=\nabla \theta(t ; .) \cdot \nu_{\left.\right|_{x y}}=0 & \text { p.p. dans } \mathbf{R}^{+} \\
\theta(0 ; .)=\theta_{0} ; V(0 ; .)=V_{0} &
\end{array}
$$

On a donc ainsi défini une solution forte du problème $\mathcal{P}^{\sigma}$.

iv) : Le théorème 1 peut s'étendre au cas d'un ouvert $\Omega$ régulier avec une donnée au bord convenable pour la température; par contre nous ne savons pas, dans un cadre plus général que celui présenté ici, obtenir les résultats $\mathrm{du}$ théorème 2 : Ceci est le fait du terme quadratique dans l'équation de Darcy-Forchheimer et du manque de régularité des solutions de cette équation (voir par exemple [11])

Afin d'alléger les démonstrations des résultats ci-dessus nous introduisons les notations complémentaires suivantes :

Pour la définition des espaces $H^{s}(\Omega)$ nous renvoyons à [1] ou [21] par exemple; on note alors : 
P. Fabrie

$$
\begin{aligned}
H^{2} & =\left\{\begin{array}{lll}
\theta, \theta \in H^{2}(\Omega) & \theta_{\mid \Gamma_{z}}=0 & \left.\frac{\partial \theta}{\partial \nu}\right|_{\Gamma_{x_{y}}^{*}}=0
\end{array}\right\} \\
H^{s} & =H^{2} \cap H^{s}(\Omega) \text { pour } s \geq 2 \\
\mathbf{H}^{s} & =W^{2} \cap\left(H^{s}(\Omega)\right)^{3}
\end{aligned}
$$

$\dot{\theta}$ et $\dot{V}$ sont respectivement les dérivées par rapport au temps de $\theta$ et $V$.

On désigne par $|\cdot|_{\sigma}$ (resp. $\|\cdot\|_{\sigma}$ ) la norme dans $L^{\sigma}$ (resp. $L^{\sigma}$ ). Le produit scalaire dans $\mathbf{R}^{n}$ est noté $(\cdot)$ et la dualité entre $H$ et $H^{\prime}$ par $\langle\cdot, \cdot\rangle$. Enfin remarquons que les conditions aux limites vérifiées par $\theta$ permettent de prendre comme norme équivalente sur $H:\|\nabla \theta\|_{2}$.

Par la suite nous utiliserons de façon essentielle le résultat suivant :

- Théorème de décomposition de Helmotz ([14], théorème 2)

Pour $1<p<\infty$ on a : $L^{p}=G_{p} \oplus W^{p}$

$$
\text { avec } \begin{aligned}
G_{p} & =\left\{U \in \mathbf{L}^{p}, \exists \pi \in W^{1, p}(\Omega), U=\nabla \pi\right\} \\
W^{p} & =\left\{U \in \mathbf{L}^{p}, \operatorname{div} U=0 \gamma_{n}(U)=0\right\} .
\end{aligned}
$$

Enfin, notons $B(V, \theta)$ l'application bilinéaire définie par $B(V, \theta)=V \cdot \nabla \theta$ et notons $b(V, \theta, \varphi)$ la forme trilinéaire définie par

$$
b(V, \theta, \varphi)=\int_{\Omega}(V \cdot \nabla \theta) \varphi d \omega .
$$

\section{Démonstration du théorème 1}

On obtient facilement les résultats d'antisymétrie et de continuité cidessous :

LEMme 1.- i) L'application $(V, \theta) \rightarrow B(V, \theta)$ est continue de $\mathbf{W}^{2} \times\left[H \cap L^{\infty}\right]$ dans $H^{\prime}$.

ii) La forme trilinéaire $b(., .,$.$) est définie et continue de$ $\mathbf{W}^{2} \times\left[H \cap L^{\infty}\right] \times\left[H \cap L^{\infty}\right]$ dans $\mathbf{R}$ et vérifie en outre :

$$
b(V, \theta, \varphi)=-b(V, \varphi, \theta) .
$$

On a, de plus, les majorations suivantes en dimension $n=3$ :

$$
\begin{gathered}
|b(V, \theta, \varphi)| \leq k_{1}\|V\|_{\mathbf{H}^{1}}\|\theta\|_{\mathbf{H}^{3 / 2}|\varphi|_{2}} \\
|b(V, \theta, \varphi)| \leq k_{2}\|V\|_{\mathbf{H}^{1}}\|\theta\|_{\mathbf{H}^{1}}\|\varphi\|_{\mathbf{H}^{1 / 2}} .
\end{gathered}
$$

Nous rappelons également le résultat de monotonie suivant : voir par exemple ([27] p. 255). 
Solutions fortes et majorations asymptotiques pour le modèle de Darcy Forchheimer

Lemme 2.- Pour tout couple $\left(V_{1}, V_{2}\right)$ de $\mathbf{R}^{n}$ on a :

$$
\begin{gathered}
\left|V_{2}-V_{1}^{\sigma+1}\right| \leq \sigma\left(\left|V_{2}\right|^{\sigma-1} V_{2}-\left|V_{1}\right|^{\sigma-1} V_{1} \cdot V_{2}-V_{1}\right) \\
\left.|| V_{2}\right|^{\sigma-1} V_{2}-\left|V_{1}\right|^{\sigma-1} V_{1}\left|\leq 3 \sigma\left(\left|V_{2}\right|+\left|V_{1}\right|\right)^{\sigma-1}\right| V_{2}-V_{1} \mid
\end{gathered}
$$

La démonstration du théorème 1 s'obtient par une méthode de point fixe; les deux lemmes ci-dessous précisent donc un théorème d'existence et d'unicité pour les deux équations découplées du problème $\mathcal{P}^{\sigma}$ et donnent des estimations à priori.

Le résultat suivant, démontré dans ([10], proposition 2) donne un théorème d'existence et d'unicité pour l'équation de l'énergie, le champ des vitesses $V$ étant donné.

LEMME 3.- Pour tout $V$ appartenant à $\mathcal{C}\left(0, T ; \mathbf{W}^{2}\right)$ et tout $\theta_{0}$ donné dans $L^{\infty}$, vérifiant de plus $0 \leq \theta+1-z / h \leq 1$ p.p, le problème :

$$
\theta(0 ; \cdot)=\theta_{0}
$$

pour tout $\varphi$ de $L^{2}(0, T ; H)$

$\int_{0}^{t}<\dot{\theta}, \varphi>d \tau+\int_{0}^{t} \int_{\Omega}(\nabla \theta \cdot \nabla \varphi) d \omega d \tau+\int_{0}^{t}\langle B(V, \theta), \varphi\rangle d \tau$ $-h^{-1} \int^{t} \int_{\Omega} v_{3} \varphi d \omega d \tau=0$

possède une unique solution dans $L^{2}(0, T ; H) \cap L^{\infty}\left(0, T ; L^{\infty}\right)$ où $\dot{\theta} \in$ $L^{2}\left(0, T ; H^{\prime}\right)$ et où $\theta$ vérifie de plus $0 \leq \theta+1-z / h \leq 1$ p.p.

De plus, il existe deux constantes $k_{1}$ et $k_{2}$ ne dépendant que de la norme de $V$ dans $\mathcal{C}\left(0, T ; \mathbf{W}^{2}\right)$ et de la mesure de $\Omega$ telles que :

$$
\|\theta\|_{L^{2}(0, T ; H)} \leq k_{1} ;\|\dot{\theta}\|_{L^{2}\left(0, T ; H^{\prime}\right)} \leq k_{2} .
$$

Par des propriétés de monotonie, de coercivité et d'hémicontinuité étudiées pour le problème stationnaire dans ([11], $1^{\text {ère }}$ partie) et des techniques très classiques ([19], chap. 2) il est facile d'obtenir le résultat ci-dessous :

Lemme 4. - Pour toute fonction $\theta$ appartenant $\dot{a}$ $\mathcal{C}\left(0, T ; L^{2}\right) \cap L^{\infty}\left(0, T ; L^{\infty}\right)$ vérifiant $0 \leq \theta+1-z / h \leq 1, \quad p . p$ et tout $V_{0}$ donné dans $\mathbf{W}^{2}$ l'équation:

$$
\begin{gathered}
V(0 ; .)=V_{0} \\
-13-
\end{gathered}
$$


pour tout $\mathbf{W}$ de $L^{\sigma+1}\left(0, T ; W^{\sigma+1}\right)$

$$
\begin{aligned}
S \int_{0}^{t} \int_{\Omega}(\dot{V} \cdot W) d \omega d \tau+\int_{0}^{t} \int_{\Omega}\left(V+J|V|^{\sigma-1} V \cdot W\right) d \omega d \tau+ & \\
& +R a^{*} \int_{0}^{t} \int_{\Omega}(k \theta \cdot W) d \omega d \tau=0
\end{aligned}
$$

possède une unique solution $V$ dans $L^{\infty}\left(0, T ; \mathbf{W}^{2}\right) \cap L^{\sigma+1}\left(0, T ; \mathbf{W}^{\sigma+1}\right)$ ò̀ $\dot{V} \in L^{1+1 / \sigma}\left(0, T ; \mathbf{W}^{1+1 / \sigma}\right)$.

De plus, il existe trois constantes $k_{3}, k_{4}, k_{5}$ ne dépendant que de $R a^{*}$ et de la mesure de $\Omega$ telles que:

$$
\|\dot{V}\|_{L^{1+1 / \sigma 1+1 / \sigma}(0, T ; W)} \leq k_{3} ; \quad\|V\|_{L^{\infty}\left(0, T ; W^{2}\right)} \leq k_{4} ; \quad\|V\|_{L^{1+\sigma 1+\sigma}(0, T ; W)} \leq k_{5} .
$$

Démontrons maintenant le théorème 1.

On considère la méthode de point fixe suivante :

$$
V^{0}=V_{0} ; \theta^{0}=\theta_{0} \text {. }
$$

Connaissant $\theta^{n}$ et $V^{n}$ on détermine $\theta^{n+1}$ et $V^{n+1}$ par :

$$
\theta_{0}^{n+1}=\theta_{0}, V_{0}^{n+1}=V_{0} .
$$

- Pour tout $\varphi$ de $L^{2}(0, T ; H)$ :

$$
\begin{aligned}
\int_{0}^{t}<\theta^{n+1}, \varphi>\mathrm{d} \tau & +\int_{0}^{t} \int_{\Omega}\left(\nabla \theta^{n+1} \cdot \nabla \varphi\right) \mathrm{d} \omega \mathrm{d} \tau+ \\
& +\int_{0}^{t}\left\langle B\left(V^{n}, \theta^{n+1}\right), \varphi>\mathrm{d} \tau-h^{-1} \int_{0}^{t} \int_{\Omega} v_{3}^{n} \varphi \mathrm{d} \omega \mathrm{d} \tau=0 .\right.
\end{aligned}
$$

- Pour tout $W$ de $L^{\sigma+1}\left(0, T ; \mathbf{W}^{\sigma+1}\right)$ :

$$
\begin{aligned}
S \int_{0}^{t} \int_{\Omega}\left(V^{n+1} \cdot W\right) \mathrm{d} \omega \mathrm{d} \tau+\int_{0}^{t} \int_{\Omega}\left(V^{n+1}\right. & \left.+J\left|V^{n+1}\right|^{\sigma-1} \cdot W\right) \mathrm{d} \omega \mathrm{d} \tau+ \\
& +R a^{*} \int_{0}^{t} \int_{\Omega}\left(k \theta^{n} \cdot W\right) \mathrm{d} \omega \mathrm{d} \tau=0 .
\end{aligned}
$$

Les lemmes 3 et 4 prouvent l'existence du couple $\left(\theta^{n+1}, V^{n+1}\right)$ et assurent l'existence de constantes indépendantes de $n$ telles que :

$$
\begin{aligned}
& \left\|\theta^{n}\right\|_{L^{\infty}\left(0, T ; L^{\infty}\right)} \leq 1 \\
& \left\|\theta^{n}\right\|_{L^{2}(0, T ; H)} \leq k_{1} \\
& \left\|\dot{\theta}^{n}\right\|_{L^{2}\left(0, T ; H^{\prime}\right)} \leq k_{2} \\
& \left\|\dot{V}^{n}\right\|_{L^{1+1 / \sigma}\left(0, T ; W^{1+1 / \sigma}\right)} \leq k_{3} \\
& \left\|V^{n}\right\|_{L^{\infty}\left(0, T ; W^{2}\right)} \leq k_{4} \\
& \left\|V^{n}\right\|_{L^{\sigma+1}\left(0, T ; W^{\sigma+1}\right)} \leq k_{5} .
\end{aligned}
$$


Solutions fortes et majorations asymptotiques pour le modèle de Darcy Forchheimer

Nous allons maintenant montrer qu'il existe un intervalle $\left(0, T_{1}\right)$ sur lequel l'application $\left(\theta^{n}, V^{n}\right) \rightarrow\left(\theta^{n+1}, V^{n+1}\right)$ est contractante. On note $\theta_{p, q}=\theta^{p}-\theta^{q}$ et $V_{p, q}=V^{p}-V^{q}$. Alors le couple $\left(\theta_{p, q}, V_{p, q}\right)$ vérifie :

$$
\begin{aligned}
& \left\{\begin{array}{l}
\forall \varphi \in L^{2}(0, T ; H) \\
\int_{0}^{t}<\theta_{p+1, q+1}, \varphi>\mathrm{d} \tau+\int_{0}^{t} \int_{\Omega}\left(\nabla \theta_{p+1, q+1} \cdot \nabla \varphi\right) \mathrm{d} \omega \mathrm{d} \tau \\
+\int_{0}^{t}<B\left(V_{p, q}, \theta^{p+1}\right), \varphi>\mathrm{d} \tau+\int_{0}^{t}<B\left(V^{q}, \theta_{p+1, q+1}\right), \varphi>\mathrm{d} \tau
\end{array}\right. \\
& \quad-h^{-1} \int_{0}^{t} \int_{\Omega} v_{p, q ; 3} \varphi \mathrm{d} \omega \mathrm{d} \tau=0 . \\
& \left\{\begin{array}{l}
\forall W \in L^{\sigma+1}\left(0, T ; \mathbf{W}^{\sigma+1}\right) \\
S \int_{0}^{t} \int_{\Omega}\left(\dot{V}_{p+1, q+1} \cdot W\right) \mathrm{d} \omega \mathrm{d} \tau+ \\
\left.\int_{0}^{t} \int_{\Omega}\left(V_{p+1, q+1}+J\left(\left|V^{p+1}\right|^{\sigma-1} V^{p+1}-\left|V^{q+1}\right|^{\sigma-1} V^{q+1}\right)\right) \cdot W\right) \mathrm{d} \omega \mathrm{d} \tau \\
+R a^{*} \int_{0}^{t} \int_{\Omega}\left(k \theta_{p, q} \cdot W\right) \mathrm{d} \omega \mathrm{d} \tau
\end{array}\right.
\end{aligned}
$$

En faisant $\varphi=\theta_{p+1, q+1}$ dans l'équation (11) et $W=V_{p+1, q+1}$ dans (12) on déduit d'après les lemmes 1,2 et 3 l'estimation suivante :

$$
\begin{aligned}
& \frac{1}{2}\left|\theta_{p+1, q+1}\right|_{2}^{2}+\int_{0}^{t}\left\|\nabla \theta_{p+1, q+1}\right\|_{2}^{2} \mathrm{~d} \tau \leq k_{6} \int_{0}^{t}\left\|V_{p, q}\right\|_{2}\left\|\nabla \theta_{p+1, q+1}\right\|_{2} \mathrm{~d} \tau \\
& -h^{-1} \int_{0}^{t}\left\|V_{p, q}\right\|_{2}\left|\theta_{p+1, q+1}\right|_{2} \mathrm{~d} \tau \\
& \frac{S}{2}\left\|V_{p+1, q+1}\right\|_{2}^{2}+\int_{0}^{t}\left\|V_{p+1, q+1}\right\|_{2}^{2} \mathrm{~d} \tau+J \sigma^{-1} \int_{0}^{t}\left\|V_{p+1, q+1}\right\|_{\sigma+1}^{\sigma+1} \mathrm{~d} \tau \\
& \leq R a^{*} \int_{0}^{t}\left|\theta_{p, q}\right|_{2}\left\|V_{p+1, q+1}\right\|_{2} \mathrm{~d} \tau .
\end{aligned}
$$

En majorant $\left|\theta_{p, q}\right|_{2}$ par $\lambda_{1}^{-1 / 2}\left\|\nabla \theta_{p, q}\right\|_{2}$ où $\lambda_{1}$ est la plus petite valeur propre de l'opérateur $-\Delta$ de domaine $H^{2}$ et en utilisant les inégalités de Young on déduit qu'il existe des constantes $k_{8}, k_{9}$ telles que :

$$
\begin{gathered}
\left|\theta_{p+1, q+1}(t)\right|_{2}^{2}+\int_{0}^{t}\left\|\nabla \theta_{p+1, q+1}(\tau)\right\|_{2}^{2} \mathrm{~d} \tau \leq k_{8} \int_{0}^{t}\left\|_{p, q}(\tau)\right\|_{2}^{2} \mathrm{~d} \tau \\
\left\{\begin{array}{c}
S\left\|V_{p+1, q+1}(t)\right\|_{2}^{2}+\int_{0}^{t}\left\|V_{p+1, q+1}(\tau)\right\|_{2}^{2} \mathrm{~d} \tau+J \sigma^{-1} \int_{0}^{t}\left\|V_{p+1, q+1}\right\|_{\sigma+1}^{\sigma+1} \mathrm{~d} \tau \\
\leq k_{9} R a^{* 2} \int_{0}^{t}\left|\theta_{p, q}(\tau)\right|_{2}^{2} \mathrm{~d} \tau
\end{array}\right.
\end{gathered}
$$


On en déduit, pour $T^{*}$ l'estimation suivante :

$$
\begin{aligned}
\sup _{t \in\left[0, T^{*}\right]}\left(\left|\theta_{p+1, q+1}(t)\right|_{2}^{2}+S\left\|V_{p+1, q+1}(t)\right\|_{2}^{2}\right) \leq \\
k_{10} T^{*} \sup _{t \in(0, T)}\left(\left|\theta_{p, q}(t)\right|_{2}^{2}+\left\|V_{p, q}(t)\right\|_{2}^{2}\right)
\end{aligned}
$$

avec $k_{10}=\operatorname{Max}\left(k_{8}, k_{9} R a^{*}\right)$.

$\mathrm{Si}$ on choisit maintenant $T_{1}=\left(2 k_{10}\right)^{-1}$ on en déduit que l'application $\left(\theta_{p}^{p}, V_{p}^{p}\right) \rightarrow\left(\theta^{p+1}, V^{p+1}\right)$ est contractante dans $L^{\infty}\left(0, T_{1} ; L^{2}\right) \times$ $L^{\infty}\left(0, T_{1} ; \mathbf{W}^{2}\right)$. Donc la suite $\left(\theta^{p}, V^{p}\right)_{p \geq 0}$ est une suite de Cauchy dans $L^{\infty}\left(0, T_{1} ; L^{2}\right) \times L^{\infty}\left(0, T_{1} ; \mathbf{W}^{2}\right)$. En utilisant les estimations (13), (14) on prouve que $\left(\theta^{p}, V^{p}\right)_{p \geq 0}$ est une suite de Cauchy dans :

$$
\left[L^{\infty}\left(0, T_{1} ; L^{2}\right) \cap L^{2}\left(0, T_{1} ; H\right)\right] \times\left[L^{\infty}\left(0, T_{1} ; \mathbf{W}^{2}\right) \cap L^{\sigma+1}\left(0, T_{1} ; \mathbf{W}^{\sigma+1}\right)\right] ;
$$

elle y possède donc une limite.

Ces résultats de convergence, complétés par des propriétés de convergences faibles résultant des estimations (5) à (10) permettent de passer à la limite dans les équations définissant $\theta^{n}$ et $V^{n}$, ce qui montre que le couple $(\theta, V)$ est solution de $\mathcal{P}^{\sigma}$ sur $\left[0, T_{1}\right]$. Comme $T_{1}$ ne dépend pas de $\theta$ et de $V$ on obtient par recollements successifs d'intervalles de longueur $T_{1}$ l'existence d'une solution sur $[0, T]$ pour tout $T$.

La différence $\theta_{1}-\theta_{2}, V_{1}-V_{2}$ de deux solutions de $\mathcal{P}^{\sigma}$ vérifie :

$$
\begin{aligned}
\left|\theta_{1}(t)-\theta_{2}(t)\right|_{2}^{2} & +\int_{0}^{t}\left\|\nabla \theta_{1}(\tau)-\nabla \theta_{2}(\tau)\right\|_{2}^{2} \\
& \leq\left|\theta_{1}(0)-\theta_{2}(0)\right|_{2}^{2}+k_{8} \int_{0}^{t}\left\|V_{1}(\tau)-V_{2}(\tau)\right\|_{2}^{2} \mathrm{~d} \tau . \\
S\left\|V_{1}(t)-V_{2}(t)\right\|_{2}^{2} & +\int_{0}^{t}\left\|V_{1}(\tau)-V_{2}(\tau)\right\|_{2}^{2}+J \sigma^{-1}\left\|V_{1}(\tau)-V_{2}(\tau)\right\|_{\sigma+1}^{\sigma+1} \mathrm{~d} \tau \\
& \leq S\left\|V_{1}(0)-V_{2}(0)\right\|_{2}^{2}+k_{9} R a^{* 2} \int_{0}^{t}\left|\theta_{1}(\tau)-\theta_{2}(\tau)\right|_{2}^{2} \mathrm{~d} \tau .
\end{aligned}
$$

En sommant ces deux inégalités et en appliquant le lemme de Gronwall (cf. [3], Annexes) à la fonction :

$$
U(t)=\left|\theta_{1}(t)-\theta_{2}(t)\right|_{2}^{2}+S\left\|V_{1}(t)-V_{2}(t)\right\|_{2}^{2}
$$

on en déduit l'unicité.

Ceci termine la démonstration du théorème 1 . 


\section{Démonstration du théorème 2}

Nous établissons maintenant le résultat principal de cet article : le théorème 2. Nous commençons par construire un espace d'approximation pour le problème $\mathcal{P}^{\sigma}$. Celui-ci nous servira également pour la discrétisation du problème. L'existence d'un tel espace d'approximation est indépendante de la géométrie de l'ouvert $\Omega$ mais sa construction effective est directement liée au fait que notre ouvert est un produit tensoriel d'intervalles. Disons simplement ici que notre espace est construit sur un sous-ensemble de fonctions périodiques.

On note :

$$
W_{i, j, k}(x, y, z)=\left\{\begin{array}{l}
i k L^{-1} h^{-1} \sin \left(i L^{-1} \pi x\right) \cos \left(j \ell^{-1} \pi y\right) \cos \left(k h^{-1} \pi z\right) \\
j k L^{-1} h^{-1} \cos \left(i L^{-1} \pi x\right) \sin \left(j \ell^{-1} \pi y\right) \cos \left(k h^{-1} \pi z\right) \\
-\left(i^{2} L^{-2}+j^{2} \ell^{-2}\right) \cos \left(i L^{-1} \pi x\right) \cos \left(j \ell^{-1} \pi y\right) \sin \left(k h^{-1} \pi z\right)
\end{array}\right.
$$

$$
V_{i, j, k}(x, y, z)=\left\{\begin{array}{l}
j \ell^{-1} \sin \left(i L^{-1} \pi x\right) \cos \left(j \ell^{-1} \pi y\right) \cos \left(k h^{-1} \pi z\right) \\
-i L^{-1} \cos \left(i L^{-1} \pi x\right) \sin \left(j \ell ^ { - 1 } \pi y \left(\cos \left(k h^{-1} \pi z\right)\right.\right. \\
0
\end{array}\right.
$$

$\theta_{i, j, k}(x, y, z)=\cos \left(i L^{-1} \pi x\right) \cos \left(j \ell^{-1} \pi y\right) \sin \left(k h^{-1} \pi z\right)$

$\bar{W}_{i, j, k}=\left[i^{2} k^{2} L^{-2} h^{-2}+j^{2} K^{2} L^{-2} h^{-2}+\left(i^{2} L^{-2}+j^{2} \ell^{-2}\right)^{2}\right]^{-1 / 2} W_{i, j, k}$

$\bar{V}_{i, j, k}=\left(j^{2} \ell^{-2}+i^{2} L^{-2}\right)^{-1 / 2} V_{i, j, k}$

$\bar{\theta}_{i, j, k}=\theta_{i, j, k}$.

Enfin on note $A_{2}$ l'opérateur de domaine $\mathbf{D}\left(A_{2}\right)=\left\{V \in \mathbf{H}^{1}, \operatorname{Rot}(\operatorname{Rot} V) \in \mathbf{W}^{2},(\operatorname{Rot} V) \Lambda \nu_{\left.\right|_{\Gamma}}=0\right\}$ défini par : pour tout $(U, V)$ de $\left.\mathbf{H}^{1}<A_{2} U, V\right\rangle=\int_{\Omega}(\operatorname{Rot} U \cdot \operatorname{Rot} V) \mathrm{d} \omega$.

LEMME 5. - Le système $\left\{\bar{w}_{i, j, k}\right\} \cup\left\{\bar{v}_{i, j, k}\right\}$ forme un système orthogonal et complet de $\mathbf{W}^{2}$ de $\mathbf{H}^{1}$ et de $\mathbf{D}\left(A_{2}\right)=\left\{V \in \mathbf{H}^{2},(\operatorname{Rot} V) \Lambda \nu_{\mid \Gamma}=0\right\}$.

Démonstration : Nous montrons d'abord que $\left\{w_{i, j, k}\right\} \cup\left\{v_{i, j, k}\right\}$ est un système orthogonal et complet de $\mathbf{W}^{2}$. 


\section{P. Fabrie}

Pour cela on rappelle le lemme de décomposition : ([14], lemme 1) $\mathbf{L}^{2}=G_{2} \oplus \mathbf{W}^{2}$. On remarque ensuite que les fonctions :

$$
\begin{aligned}
& \sin \left(i L^{-1} \pi x\right) \cos \left(j \ell^{-1} \pi y\right) \cos \left(k h^{-1} \pi z\right) e_{1} \\
& \cos \left(i L^{-1} \pi x\right) \sin \left(j \ell^{-1} \pi y\right) \cos \left(k h^{-1} \pi z\right) e_{2} \\
& \cos \left(i L^{-1} \pi x\right) \cos \left(j \ell^{-1} \pi y\right) \sin \left(k h^{-1} \pi z\right) e_{3}
\end{aligned}
$$

où $\left(e_{1}, e_{2}, e_{3}\right)$ est la base canonique de $\mathbf{R}^{3}$ forme un système orthogonal et complet de $\mathbf{L}^{2}$. On décompose ensuite chacune de ces fonctions en une somme d'un gradient et d'une combinaison linéaire de $\bar{v}_{i, j, k}, \bar{w}_{i, j, k}$; le gradient s'écrivant : $\alpha \nabla \pi_{i, j, k}$ où $\pi_{i, j, k}=\cos \left(i L^{-1} \pi x\right) \cos \left(j L^{-1} \pi y\right) \cos \left(k h^{-1} \pi z\right)$. On en déduit alors la densité de $\left\{\bar{w}_{i, j, k}\right\} \cup\left\{\bar{v}_{i, j, k}\right\}$ dans $\mathbf{W}^{2}$. Enfin on note que $A_{2}$ est un opérateur auto-adjoint non borné sur $\mathbf{H}^{1}$.

Il s'en suit alors que l'ensemble des fonctions propres de $A_{2}$ forme un ensemble complet et orthogonal dans $\mathbf{W}^{2}$, dans $\mathbf{H}^{1}$, et dans $\mathbf{D}\left(A_{2}\right)$. On vérifie que les fonctions $\left\{\bar{w}_{i, j, k}\right\} \cup\left\{\bar{v}_{i, j, k}\right\}$ sont fonctions propres de $A_{2}$ et comme d'après la première partie $\left\{\bar{w}_{i, j, k}\right\} \cup\left\{\bar{v}_{i, j, k}\right\}$ est dense dans $\mathbf{W}^{2}$; on a obtenu ainsi toutes les fonctions propres de $A_{2}$ dans $\mathbf{W}^{2}$. Ceci termine la démonstration du lemme 5 .

LEMME 6. - Le système $\left\{\theta_{i, j, k}\right\}$ forme un système orthogonal et complet de $L^{2}$, de $H$ et de $H^{2}$.

Cette démonstration est classique, voir par exemple ([20], p. 44) les fonctions $\bar{\theta}_{i, j, k}$ étant les fonctions propres de l'opérateur de Laplace, de domaine $\mathbf{H}^{2}$.

On note ici $\mathbf{W}_{\mu}^{2}$ l'espace vectoriel engendré par les fonctions propres de l'opérateur $A_{2}$ de domaine $\mathbf{D}\left(A_{2}\right)$ associé aux $\mu$ premières valeurs propres, $H_{\mu}$ l'espace vectoriel engendré par les fonctions propres de l'opérateur de Laplace de domaine $H^{2}$ associées aux $\mu$ premières valeurs propres.

$\mathbf{P}_{\mu}$ désigne alors la projection orthogonale de $\mathbf{W}^{2}$ sur $\mathbf{W}_{\mu}^{2}$ et $P_{\mu}$ la projection orthoganale de $L^{2}$ sur $H_{\mu}$.

Dans [11] nous avons montré que la solution $V$ de l'équation:

$$
\left\{\begin{array}{l}
V+|V|^{\sigma-1} V+\nabla \pi=f \\
\operatorname{div} V=0 \\
V \cdot \nu_{\left.\right|_{\Gamma}}=0
\end{array}\right.
$$

appartient à $\left(H_{\text {loc }}^{1}(\Omega)\right)^{3}$ dès que $f$ appartient à $\left(H_{\text {loc }}^{1}(\Omega)\right)^{3}$. 
Solutions fortes et majorations asymptotiques pour le modèle de Darcy Forchheimer

Nous avons ici un résultat analogue pour la solution de l'équation (1.2). Cependant, vu la géométrie particulière de l'ouvert $\Omega$ et les conditions aux limites vérifiées par $V$ et $\theta$ on peut donner ici un résultat de régularité globale plus direct.

Nous obtenons le résultat suivant :

LeMme 7. - Soit $\theta$ un élément de $L^{2}(0, T ; H)$ et $V_{0}$ élément de $\mathbf{H}^{1}$. Alors la solution $V$ de l'équation:

$$
\left\{\begin{array}{l}
V(0 ; .)=V_{0} \\
S \frac{\partial V}{\partial t}+V+J|V|^{\sigma-1} V+\nabla \pi=R a^{*} k \theta
\end{array}\right.
$$

appartient $\grave{a} L^{\infty}\left(0, T ; \mathbf{H}^{1}\right) \cap L^{\sigma+1}\left(0, T ; \mathbf{W}^{\sigma+1}\right)$ et on a l'estimation :

$$
\|V\|_{L^{\infty}\left(0, T, \mathbf{H}^{1}\right)}^{2} \leq S^{-1} R a^{*} \int_{0}^{T}\|\nabla \theta\|_{2}^{2} d \tau+\left\|V_{0}\right\|_{\mathbf{H}^{1}}^{2} .
$$

Démonstration : L'unicité se déduit du lemme 4; montrons la régularité énoncée.

On cherche $V_{\mu}$ appartenant à $\mathbf{W}_{\mu}^{2}$ solution de :

$$
\begin{aligned}
& V_{\mu}(0 ; .)=\mathbf{P}_{\mu}\left(V_{0}\right) \\
& S \frac{\partial V_{\mu}}{\partial t}+V_{\mu}+J \mathbf{P}_{\mu}\left(\left|V_{\mu}\right|^{\sigma-1} V_{\mu}\right)=R a^{*} P_{\mu}(k \theta)
\end{aligned}
$$

en multipliant cette équation par $-\Delta V_{\mu}$, une intégration par parties donne :

$$
\begin{array}{r}
\frac{S}{2} \frac{\mathrm{d}}{\mathrm{dt}}\left\|\nabla V_{\mu}\right\|_{2}^{2}+\left\|\nabla V_{\mu}\right\|_{2}^{2}+J \int_{\Omega}\left(\nabla\left(\left|V_{\mu}\right|^{\sigma-1} V_{\mu}\right) \cdot \nabla V_{\mu}\right) \mathrm{d} \omega \\
\leq R a^{*}\|\nabla \theta\|_{2}\left\|\nabla V_{\mu}\right\|_{2} .
\end{array}
$$

Le terme intégral peut se transformer de la manière suivante :

$$
\begin{gathered}
\int_{\Omega}\left(\nabla\left(\left|V_{\mu}\right|^{\sigma-1} V_{\mu}\right) \cdot \nabla V_{\mu}\right) \mathrm{d} \omega=\int_{\Omega}\left|V_{\mu}\right|^{\sigma-1}\left(\nabla V_{\mu} \cdot \nabla V_{\mu}\right) \mathrm{d} \omega \\
=\int_{\Omega}|V|^{\sigma-3} \sum_{i=1}^{3}\left(\sum_{k=1}^{3} \frac{\partial V_{k}}{\partial x_{i}} V_{k}\right)^{2} \mathrm{~d} \omega .
\end{gathered}
$$

d'où l'on déduit que $\int_{\Omega}\left(\nabla\left|V_{\mu}\right|^{\sigma-1} V_{\mu} \cdot \nabla V_{\mu}\right) \mathrm{d} \omega$ est positif. 
Donc, d'après l'inégalité (16) on a, en appliquant l'inégalité de Young :

$$
S \frac{\mathrm{d}}{\mathrm{dt}}\left\|\nabla V_{\mu}\right\|_{2}^{2}+\left\|\nabla V_{\mu}\right\|_{2}^{2} \leq R a^{*}\|\nabla \theta\|_{2}^{2} .
$$

Une intégration en temps donne alors :

$$
\left\|\nabla V_{\mu}(t)\right\|_{2}^{2} \leq\left\|\nabla V_{\mu}(0)\right\|_{2}^{2}+S^{-1} R a^{*} \int_{0}^{t}\|\nabla \theta\|_{2}^{2} \mathrm{~d} \tau .
$$

Par ailleurs, on vérifie que $\left\|\nabla V_{\mu}(0)\right\|_{2} \leq\|\nabla V(0)\|_{2}$. Il existe donc un élément $\widetilde{V}$ de $L^{\infty}\left(0, T ; \mathbf{H}^{1}\right)$ limite faible de la suite $\left(V_{\mu}\right)_{\mu}$. D'autre part $\widetilde{V}$ vérifie pour tout $W$ de $\mathbf{W}_{\mu}^{2}$ :

$$
\left.S \int_{\Omega}\left(\frac{\partial \tilde{V}}{\partial t} \cdot W\right) \mathrm{d} \omega+(\tilde{V} \cdot W)+(\chi \cdot W)\right) \mathrm{d} \omega=R a^{*} \int_{\Omega}(k \theta \cdot W) \mathrm{d} \omega
$$

où $\chi$ est la limite faible de $\left|V_{\mu}\right|^{\sigma-1} V_{\mu}$ dans $\mathbf{L}^{1+1 / \sigma}$. Comme $\underset{\mu \geq 1}{U}\left(W_{\mu}^{2}\right)$ est dense dans $\mathbf{D}\left(A_{2}\right)$ qui s'injecte continuement dans $\mathbf{W}^{\sigma+1}$ on en déduit que la relation (20) est valable pour tout $W$ de $\mathbf{W}^{\sigma-1}$. Enfin, par un argument classique de monotonie, on montre que $\chi=|\dot{\widetilde{V}}|^{\sigma-1} \widetilde{V}$ d'où l'on déduit $\widetilde{V}=V$. Enfin, comme la norme $\|\nabla \cdot\|_{2}$ est faiblement semi-continue, on déduit de l'estimation (19) l'inégalité (16). Ceci termine la démonstration du lemme 7 .

LeMme 8.- On suppose que $V$ appartient $\grave{a} L^{\infty}\left(0, T ; \mathbf{H}^{1}\right)$ et que $\theta_{0}$ appartient à $L^{\infty} \cap H$, vérifiant $0 \leq \theta_{0}+1-z / h \leq 1$ p.p. . Alors la solution de l'équation:

$$
\left\{\begin{array}{l}
\theta(0 ; .)=\theta_{0} \\
\dot{\theta}-\Delta \theta+B(V, \theta)-h^{-1} v_{3}=0
\end{array}\right.
$$

appartient $\grave{a} L^{\infty}(0, T ; H) \cap L^{2}\left(0, T ; H^{2}\right)$ et il existe une constante $k_{13}$ dépendant de la norme de $V$ dans $L^{\infty}\left(0, T ; \mathbf{H}^{1}\right)$ et de $T$ telle que :

$$
\|\theta\|_{L^{\infty}(0, T ; H)} \leq k_{13}
$$

Démonstration : On considère le problème approché suivant : Trouver $\theta_{n}$ dans $H_{n}$ solution de :

$$
\dot{\theta}_{n}-\Delta \theta_{n}+P_{n}\left(V \cdot \nabla \theta_{n}\right)-h^{-1} P_{n}\left(v_{3}\right)=0 .
$$


Solutions fortes et majorations asymptotiques pour le modèle de Darcy Forchheimer

Pour obtenir une estimation sur $\left(\nabla \theta_{n}\right)$ on multiplie l'équation ci-dessus par $-\Delta \theta_{n}$. On obtient d'après l'inégalité (2) du lemme 1 :

$$
\frac{1}{2} \frac{\mathrm{d}}{\mathrm{dt}}\left\|\nabla \theta_{n}\right\|_{2}^{2}+\left|\Delta \theta_{n}\right|_{2}^{2} \leq k_{1}\|V\|_{H^{1}}\left\|\nabla \theta_{n}\right\|_{2}^{1 / 2}\left|\Delta \theta_{n}\right|_{2}^{1 / 2}+h^{-1}\|V\|_{2}\left|\Delta \theta_{n}\right|_{2} .
$$

D'où en utilisant les inégalités de Young :

$$
\frac{\mathrm{d}}{\mathrm{dt}}\left\|\nabla \theta_{n}\right\|_{2}^{2}+\left|\Delta \theta_{n}\right|_{2}^{2} \leq k_{14}\|V\|_{H^{1}}^{4}\left\|\nabla \theta_{n}\right\|_{2}^{2}+\|V\|_{2}^{2} .
$$

Le lemme de Gronwall permet de conclure : pour $t \in[0, T]$

$$
\left\|\nabla \theta_{n}(t)\right\|_{2}^{2} \leq\left(\left\|\nabla \theta_{n}(0)\right\|_{2}^{2}+T\|V\|_{L^{\infty}\left(0, T ; W^{2}\right)}^{2}\right) \exp \left(k_{14} \int_{0}^{T}\|V(\tau)\|_{H^{1}}^{4} \mathrm{~d} \tau\right) .
$$

En remarquant que $\theta_{n}$ converge dans $L^{\infty}(0, T ; H)$ faiblement vers $\theta$, unique solution de (21) on obtient le résultat énoncé. Ceci termine la démonstration du lemme 8.

Maintenant que nous avons obtenu un résultat de régularité nous établissons les estimations asymptotiques du théorème 2 . Pour faciliter la lecture de cette démonstration nous en présentons chaque étape sous la forme d'un lemme.

Lemme 9. - Pour $\theta$ appartenant à $L^{\infty}\left(0, T ; L^{\infty}\right)$, vérifiant $0 \leq \theta+1-$ $z / h \leq 1 \quad p . p$, la solution $V$ de l'équation (15) vérifie :

$$
V \in L^{\infty}\left(\mathbf{R}^{+} ; \mathbf{W}^{2}\right)
$$

et on a l'estimation suivante :

$$
\|V(t)\|_{2}^{2} \leq e^{-s^{-1} t}\|V(0)\|_{2}^{2}+k_{15} R a^{*}
$$

où $k_{15}$ ne dépend que de la mesure de $\Omega$.

Démonstration : En multipliant l'équation (12) par $V$ et en intégrant sur $\Omega$ on obtient :

$$
\frac{S}{2} \frac{\mathrm{d}}{\mathrm{dt}}\|V\|_{2}^{2}+\left\|V_{2}^{2}+J\right\| V\left\|_{\sigma+1}^{\sigma+1} \leq R a^{*}|\theta|_{2}\right\| V \|_{2}
$$

comme $\theta$ est borné par 1 en norme $L^{\infty}\left(\mathbf{R}^{+} \times \Omega\right)$ on a $|\theta|_{2} \leq[\operatorname{Mes}(\Omega)]^{1 / 2}$. D'où en utilisant l'inégalité de Young :

$$
S \frac{\mathrm{d}}{\mathrm{dt}}\|V\|^{2}+\|V\|^{2} \leq R a^{* 2}|\Omega|
$$


En intégrant sur l'intervalle $(0, t)$ on obtient :

$$
e^{s^{-1} t}\|V(t)\|_{2}^{2} \leq\|V(0)\|_{2}^{2}+\left(\int_{0}^{t} e^{s^{-1} \tau} \mathrm{d} \tau\right) \cdot S^{-1} R a^{* 2}|\Omega|
$$

(22) s'en suit immédiatement.

LEMME 10.- Soit $V$ donné vérifiant (22) et $\theta$ solution de (21) alors il existe une constante $k_{16}$ ne dépendant que de $|\Omega|,\left\|V_{0}\right\|_{2}$ et de $R a^{*}$ telle que :

$$
\forall(s, t) \quad s \leq t \leq s+1, \int_{s}^{t}\|\nabla \theta(\tau)\|_{2}^{2} d \tau \leq k_{16}
$$

Démonstration : On multiplie l'équation (4.1) par $\theta$ et on intègre sur $\Omega$; on obtient :

$$
\frac{1}{2} \frac{\mathrm{d}}{\mathrm{dt}}|\theta|_{2}^{2}+\|\nabla \theta\|_{2}^{2} \leq h^{-1}\|V\|_{2}|\theta|_{2} .
$$

Comme $\theta$ est majorée par un en norme $L^{\infty}\left(\mathbf{R}^{+} \times \Omega\right)$ on déduit d'après le lemme 9 qu'il existe une constante $k_{17}$ ne dépendant que de $R a^{*}$ et de $\Omega$ telle que :

$$
\frac{1}{2} \frac{\mathrm{d}}{\mathrm{dt}}|\theta|_{2}^{2}+\|\nabla \theta\|_{2}^{2} \leq k_{17}
$$

en intégrant sur l'intervalle $(s, t)$ on obtient :

$$
\frac{1}{2}|\theta(t)|_{2}^{2}+\int_{s}^{t}\|\nabla \theta(\tau)\|^{2} \mathrm{~d} \tau \leq|\theta(s)|_{2}^{2}+k_{17}(t-s) .
$$

Comme $\theta$ est majorée par un en norme $L^{\infty}\left(\mathbf{R}^{+} \times \Omega\right):|\theta(s)|_{2}^{2} \leq|\Omega|$ et puisque $0 \leq t-s \leq 1$, on a :

$$
\int_{s}^{t}\|\nabla \theta(\tau)\|^{2} \mathrm{~d} \tau \leq|\Omega|+k_{17}=k_{16}
$$

LEMME 11. - Soit $V_{0}$ donné dans $\mathbf{H}^{1}$ et $\boldsymbol{\theta}$ vérifiant (23) alors la solution $V$ de (15) appartient $\grave{a} L^{\infty}\left(\mathbf{R}^{+}, \mathbf{H}^{\mathbf{1}}\right)$.

Démonstration: On reprend l'inégalité (18) obtenue pour les solutions approchées $V_{\mu}$ que l'on peut aussi écrire pour $V$

$$
\frac{\mathrm{d}}{\mathrm{dt}}\|\nabla V\|_{2}^{2}+S^{-1}\|\nabla V\|_{2}^{2} \leq R a^{*} S^{-1}\|\nabla \theta\|_{2}^{2} .
$$


Solutions fortes et majorations asymptotiques pour le modèle de Darcy Forchheimer

En multipliant les deux membres de cette inégalité par $e^{s^{-1} \tau}$ on obtient :

$$
\frac{\mathrm{d}}{\mathrm{dt}}\left(e^{s^{-1} \tau}\|\nabla V(\tau)\|_{2}^{2}\right) \leq R a^{*} S^{-1} e^{s^{-1} \tau}\|\nabla \theta(\tau)\|_{2}^{2}
$$

En intégrant cette inégalité sur l'intervalle $(s, t)$ où $s \leq t \leq s+1$, on a :

$$
\begin{aligned}
e^{s^{-1}}\|\nabla V(t)\|_{2}^{2} & \leq e^{s^{-1} s}\|\nabla V(s)\|_{2}^{2}+R a^{*} S^{-1} \int_{s}^{t} e^{s^{-1} \tau}\|\nabla \theta(\tau)\|_{2}^{2} \mathrm{~d} \tau \\
& \leq e^{s^{-1} s}\|\nabla V(s)\|_{2}^{2}+R a^{*} S^{-1} e^{s^{-1} t} \int_{s}^{t}\|\nabla \theta(\tau)\|_{2}^{2} \mathrm{~d} \tau
\end{aligned}
$$

D'après l'estimation (23) on obtient :

$$
\|\nabla V(t)\|_{2}^{2} \leq e^{s^{-1}(s-t)}\left(\|\nabla V(s)\|_{2}^{2}\right)+R a^{*} S^{-1} k_{16}
$$

Puisque d'après le lemme $7, V$ appartient à $L^{\infty}\left(0, T ; \mathbf{H}^{1}\right)$ on peut supposer sans perte de généralité que $t$ est supérieur à 1 . En écrivant alors l'inégalité précédente pour $s=t-1$, on obtient :

$$
\|\nabla V(t)\|_{2}^{2} \leq e^{-s^{-1}}\|\nabla V(t-1)\|_{2}^{2}+k_{18} .
$$

Soit $E(t)$ la partie entière de $t$. Une récurrence montre alors que :

$$
\|\nabla V(t)\|_{2}^{2} \leq e^{-E(t) s^{-1}}\|\nabla V(t-E(t))\|_{2}^{2}+k_{18} \sum_{k=0}^{E(t)} e^{-s^{-1} k}
$$

En majorant $\sum_{k=0}^{E(t)} e^{-s^{-1} k}$ par $\frac{1}{1-e^{-s^{-1}}}, \quad$ on obtient :

$$
\|\nabla V(t)\|_{2}^{2} \leq e^{-E(t) S^{-1}}\|\nabla V(t-E(t))\|_{2}^{2}+k_{19} .
$$

En remarquant que $0 \leq t-E(t)<1$ et en utilisant (16), on a :

$$
\|\nabla V(t)\|_{2}^{2} \leq e^{-E(t) S^{-1}}\left(\left\|\nabla V_{0}\right\|_{2}^{2}+R a^{*} \int_{0}^{1}\|\nabla \theta(\tau)\|_{2}^{2} \mathrm{~d} \tau\right)+k_{19}
$$

D'où :

$$
\|\nabla V(t)\|_{2}^{2} \leq k_{20}
$$




\section{P. Fabrie}

Nous pouvons maintenant terminer la démonstration du théorème. On multiplie l'équation (1.1) par $-\Delta \theta$, ce qui est loisible d'après le lemme 8 et on intègre sur l'ouvert $\Omega$. On obtient l'inégalité de l'énergie suivant :

$$
\frac{1}{2} \frac{\mathrm{d}}{\mathrm{d} t}\|\nabla \theta\|_{2}^{2}+\left.\left|\Delta \theta \|_{2}^{2}+\right| \Delta \theta\right|_{2} ^{2} \leq\left|\int_{\Omega}(V \cdot \nabla \theta) \Delta \theta \mathrm{d} \omega\right|+h^{-1}\left|\int_{\Omega} v_{3} \cdot \Delta \theta \mathrm{d} \omega\right| .
$$

D'après l'inégalité (2) on a, par interpolation :

$$
\frac{1}{2} \frac{\mathrm{d}}{\mathrm{d} t}\|\nabla \theta\|_{2}^{2}+|\Delta \theta|_{2}^{2} \leq k_{21}\|V\|_{\mathbf{H}^{1}}\|\nabla \theta\|_{2}^{1 / 2}|\Delta \theta|_{2}^{1 / 2}+h^{-1}\|V\|_{2}|\Delta \theta|_{2} .
$$

D'après le lemme 11, les inégalités de Young permettent d'écrire qu'il existe des constantes $k_{22}$ et $k_{23}$ telles que :

$$
\frac{\mathrm{d}}{\mathrm{dt}}\|\nabla \theta\|_{2}^{2}+|\Delta \theta|_{2}^{2} \leq k_{22}\|\nabla \theta\|_{2}^{2}+k_{23} .
$$

En intégrant cette inégalité sur l'intervalle $(s, t), s \leq t \leq s+1$, on obtient :

$$
\|\nabla \theta(t)\|_{2}^{2}+\int_{s}^{t}|\Delta \theta(\tau)|^{2} d \tau \leq k_{22} \int_{s}^{t}\|\nabla \theta(\tau)\|^{2} d \tau+k_{23}+\|\nabla \theta(s)\|^{2} .
$$

D'où, d'après l'inégalité (23) on a :

$$
\|\nabla \theta(t)\|^{2} \leq k_{24}+\|\nabla \theta(s)\|^{2} .
$$

En intégrant cette identité dans la variable $s$ sur l'intervalle $(t-1, t)$ on obtient :

$$
\|\nabla \theta(t)\|^{2} \leq k_{25}
$$

Ceci termine la démonstration du théorème 2 .

Remerciements :

L'auteur tient à remercier le Professeur Jean Claude Saut pour l'avoir encouragé à étudier ce problème, ainsi que le Professeur Jacques Simon pour ses remarques constructives. 
Solutions fortes et majorations asymptotiques pour le modèle de Darcy Forchheimer

\section{Bibliographie}

[1] Adams (R.A.). - Sobolev space; Academic press, New-York, 1975.

[2] Agmon (S.). - Lectures on elliptic boundary value problem; Van NostrandReinhold Princeton New-Jersey, 1965.

[3] BREzis (H.).- Opérateurs maximaux monotones et semi-groupes de contraction dans les espaces de Hilbert; North-Holland, Amsterdam, 1973.

[4] Caltagirone (J.P.). - Thermoconvective instabilities in a horizontal porous Layer, J. Fluid Mech. t. 72, 1975.

[5] Caltagirone (J.P.).-Convection in porous medium. Convective transport and instability phenomena (ed. G. Braun); p. 199-232, Karlsruhe.

[6] Caltagirone (J.P.), Coulaud (O.), Morel (P.). - Effets non linéaires dans les écoulements en milieu poreux C.R. Acad. Sci. Paris série II t. 302, $\mathrm{n}^{\circ} 6$ p. 263-266, 1986.

[7] Caltagirone (J.P.), Fabrie (P.). - Convection naturelle en milieu poreux à grands nombres de Rayleigh, Partie I : modèle de Darcy. Rapport interne, Université de Bordeaux I; à paraître dans European Journal of Mechanics (1989).

[8] Combarnous (M.). - Thèse Paris 1975.

[9] Duvaut (G.), Lions (J.L.). - Les inéquations en mécanique et physique. Dunod, 1972.

[10] FABRIE (P.). - Solutions fortes et comportement asymptotique pour un modèle de convection naturelle en milieu poreux Acta Appl. Math., vol. 7 p. 49-77 1986.

[11] FABRIE (P.). - Régularité de la solution de l'équation de Darcy-Forchheimer. Rapport interne, Université de Bordeaux $I \mathrm{n}^{\circ} 8610$; à paraître à Non Linear Analysis (1989).

[12] Fabrie (P.), Caltagirone (J.P.). - Convection naturelle en milieu poreux à grands nombres de Rayleigh, Partie II : modèle de Darcy-Forchheimer. Rapport interne, Université de Bordeaux I; à paraître dans European Journal of Mechanics (1989).

[13] Foias (C.), Temam (R.). - Remarques sur les équations de Navier-Stokes stationnaires et les phénomènes successifs de bifurcation. Ann. Sc. Norm. Super. Pisa série IV vol. 5.1, 1978.

[14] Fujiwara (D.) Morimoto (H.). - An $L_{r}$ theorem of the Helmotz decomposition of vector fields-J. Tokyo Sect. 1, vol. 24 p. 685-700 1971.

[15] GLowinski (R.), Marroco (A.). - Sur l'approximation par éléments finis d'ordre un et la résolution... RAIRO, vol. 2 p. 41-76 1975 .

[16] GRISvard (P.). - Behaviour of the solution of an elliptic boundary value problem in polygonal or polyhedral domain, Numerical solution of partial differential equations III, Synspade 1975, Academic Press, New-York, San Francisco, London, 1976.

[17] HiLle -. - Functional analysis and semi-group.A.M.S., 1948. 


\section{P. Fabrie}

[18] LADYzhensKaya (O.A.). - The mathematical theory of viscous incompressible flow, $2^{\text {nd }}$ edn. Gordon and Beach, New-York, 1969.

[19] Lions (J.L.). - Quelques méthodes de résolution de problèmes aux limites non linéaires. Dunod Paris 1969.

[20] Lrons (J.L.). - Lectures on elliptic partial differential equations. Tata Institute of Fundamental Research, Bombay 1957.

[21] Lions (J.L.), Magenes (E). - Problèmes aux limites non homogènes et applications vol. 1 et 2 Dunod, Paris 1968.

[22] MC CRACKen (M.). - The stokes equations in $L_{p}$; Thesis University of California, Berkeley.

[23] Nields (D.A.), Joseph (D.D.). - Effects of quadratic deag on convection in a satured porous medium. Communication personnelle de D.D. JOSEPH- et Phys. Fluids 28 (3), 1985.

[24] NeCAS (J.). - Les méthodes directes en théorie des équations elliptiques. Masson, 1967.

[25] Politano (H.). - Convection en milieu poreux : Étude de la dynamique sur des modèles à petites dimensions de codimension 1 ou 2. (Thèse Nice 1984).

[26] RILEY (D.S.), REes (D.A.S.). - Non Darcy natural convection from arbitrarily inclined heated surfaces in saturated porous media J. Mech. Appl. Math. vol. 38 p. 277-295 1985.

[27] Simon (J.). - Régularité de la solution d'un problème aux limites non linéaires Ann. Fac. Sci Toulouse, vol. 33 p. 247-274 1981.

[28] Simon (J.).-Caractérisation d'espaces fonctionnels. Bolletino UMI (5) 15.B, p. 687-714 1978 .

[29] Simon (J.). - Régularité de la composée de deux fonctions et applications. Bolletino UMI 16.B, p. 501-522 1979.

[30] Temam (R.). - Navier-Stokes equations and nonlinear functional analysis. CBMSNSF regional conference series in applied mathematic, New-York, Philadelphie, SIAM J. ${ }_{198}$.

[31] TOLKSDORF (P.). - Regularity for a more general class of quasilinear elliptic equations; J. Differ. Equations, vol. 51, p. 126-250 1984 .

[32] Triebel (H.). - Interpolation theory function spaces, differential operator, NorthHolland, New-York, 1978. 